\title{
Prevalence of Hyperaldosteronism in Primary Care Patients with Resistant Hypertension
}

\author{
Guido Schmiemann, MD, MPH, Klaus Gebhardt, MD, Eva Hummers-Pradier, MD \\ and Günther Egidi, MD
}

Introduction: Because hyperaldosteronism is the most common curable reason for secondary hypertension, screening is recommended. However, prevalence among general practice patients and feasibility of screening is still unclear. A design to assess prevalence in general practice and barriers against screening was created.

Methods: This was an open, observational pilot study and focus group. In 2 general practices, all patients with arterial hypertension were included. Those with resistant hypertension $(>140 / 90 \mathrm{~mm} \mathrm{Hg}$ and taking $\geq 3$ antihypertensive drugs) were eligible for screening. The design and feasibility of the study were discussed in a focus group of experienced general practitioners.

Results: Of 3107 patients visiting the practices, 564 were diagnosed as having arterial hypertension. Seventy-nine fulfilled criteria for resistant hypertension. Aldosterone:renin ratio (ARR) could be measured in 63 of those patients. Withdrawal of $\beta$-blocker was feasible in 34 of the 63 with measurable ARR. ARR was positive in 15, and in 3 of those 15 with positive ARR, it was caused by elevated aldosterone levels. Focus group discussion revealed barriers and concerns regarding organizational, financial, and practical aspects of a systematic screening.

Conclusions: Screening for hyperaldosteronism in general practice seems possible in selected patients, but not in a systematic way. Barriers against systematic screening were a necessity for $\beta$-blocker cessation as well as structural prerequisites for patient identification. (J Am Board Fam Med 2012;25: 98-103.)

Keywords: Aldosterone, Hypertension, Primary Health Care, Renin, Screening

Secondary hypertension is common among patients with resistant hypertension, but the overall prevalence still is unknown. ${ }^{1}$ Because hyperaldosteronism (HA) is supposed to be the most common, curable reason for secondary hypertension, a debate about the effectiveness of screening for HA in general practice has been ongoing for some time. ${ }^{2,3}$ Important aspects of this debate are the issues of whom and how to screen. A reason for screening

This article was externally peer reviewed.

Submitted 24 March 2011; revised 8 July 2011; accepted 11 July 2011.

From the Department of General Practice, Medical School Hannover, Germany (EH-P); General Practice, Bremen, Germany (KG, GE); Department of Health Services Research University Bremen, Germany (GS).

Funding: none.

Conflict of interest: none declared.

Corresponding author: Dr. Guido Schmiemann, Department for Health Services Research University Bremen, Grazer Str. 4,28359 Bremen, Germany (E-mail: schmiemann@uni-bremen.de).
(Why should we screen?) is the increased cardiovascular risk among those with $\mathrm{HA},{ }^{4}$ with specific treatment options such as spironolactone or (partial) adrenalectomy in case of adenomas. The last option has proven to be cost-effective. ${ }^{3}$ The debate about the target group (Who should be screened?) still is ongoing. The formerly used approach of screening only those with hypokalemia is insufficient because the majority of patients with HA also are normokalemic. ${ }^{2}$ Introduction of sensitive laboratory tests such as the aldosterone/renin ratio (ARR) has led to an increase in reported cases. However, because specificity is low, a second test for confirmation (eg, the acute saline loading test) is advocated by existing guidelines ${ }^{4}$ but has been questioned by some authors. ${ }^{5}$ Because prevalence rises with increasing blood pressure, ${ }^{6}$ screening is recommended for those with resistant hypertension. ${ }^{4}$ But in which group do prevalence rates justify recommendation for active screening? The range of 
elevated ARR in primary care is 7.5 (patients with mild hypertension) to $32 \%$ (outpatient clinic for patients with drug-resistant hypertension); the range is $0.7 \%$ to $12 \%$ for patients with confirmed HA. ${ }^{7,8}$ Differences are explained partly by methodological aspects (How should we screen?). Among these differences are cutoff values for laboratory testing or different protocols for cessation of antihypertensive treatment. Regarding cessation of antihypertensive treatment, consensus has been reached for suspending the use of spironolactone and $\beta$-blockers. ${ }^{9}$ In our study we wanted to assess (1) the feasibility of a systematic screening at general practice level, and (2) the prevalence of HA in selected primary care patients with a view of conducting an adequately powered larger study.

\section{Methods}

The study was designed as a prospective, open, observational study. Ethical approval was given by the local ethics committee. Inclusion criteria was blood pressure $>140 / 90 \mathrm{~mm} \mathrm{Hg}$ (average of 3 measures by doctors, nurses, patients themselves, or 24-hour monitoring) while the patient is taking $\geq 3$ antihypertensive drugs. Compliance with medication regime was rated by the treating general practitioner (GP) on the basis of the prescription records. Exclusion criteria were pregnancy, known secondary hypertension, and/or necessity of spironolactone medication according to treating GP.

All patients who attended 2 urban group practices in northern Germany between July and December 2008 were included. The flow of patients is presented in Figure 1.

In eligible patients, ARR was measured after cessation of $\beta$-blockers for 2 weeks (cessation of spironolactone for 4 weeks), if clinically feasible. All other antihypertensive drugs were continued. Pre-existing hypokalemia was corrected, and blood samples for measurement of aldosterone and renin were taken at mid-morning (after 5 min of sitting quietly) and were transported according to laboratory requirements.

Aldosterone was measured as plasma aldosterone concentration $(40-310 \mathrm{pg} / \mathrm{mL}$ supine); renin was measured as plasma renin concentration (2.64$27.7 \mathrm{pg} / \mathrm{mL}$ supine). The ARR reference was $<45$,

Figure 1. Patient flow. *Cessation of ß blocker/spironolactone not possible because of organizational barriers, patients' characteristics and other barriers. ARR, aldosterone-renin ratio.

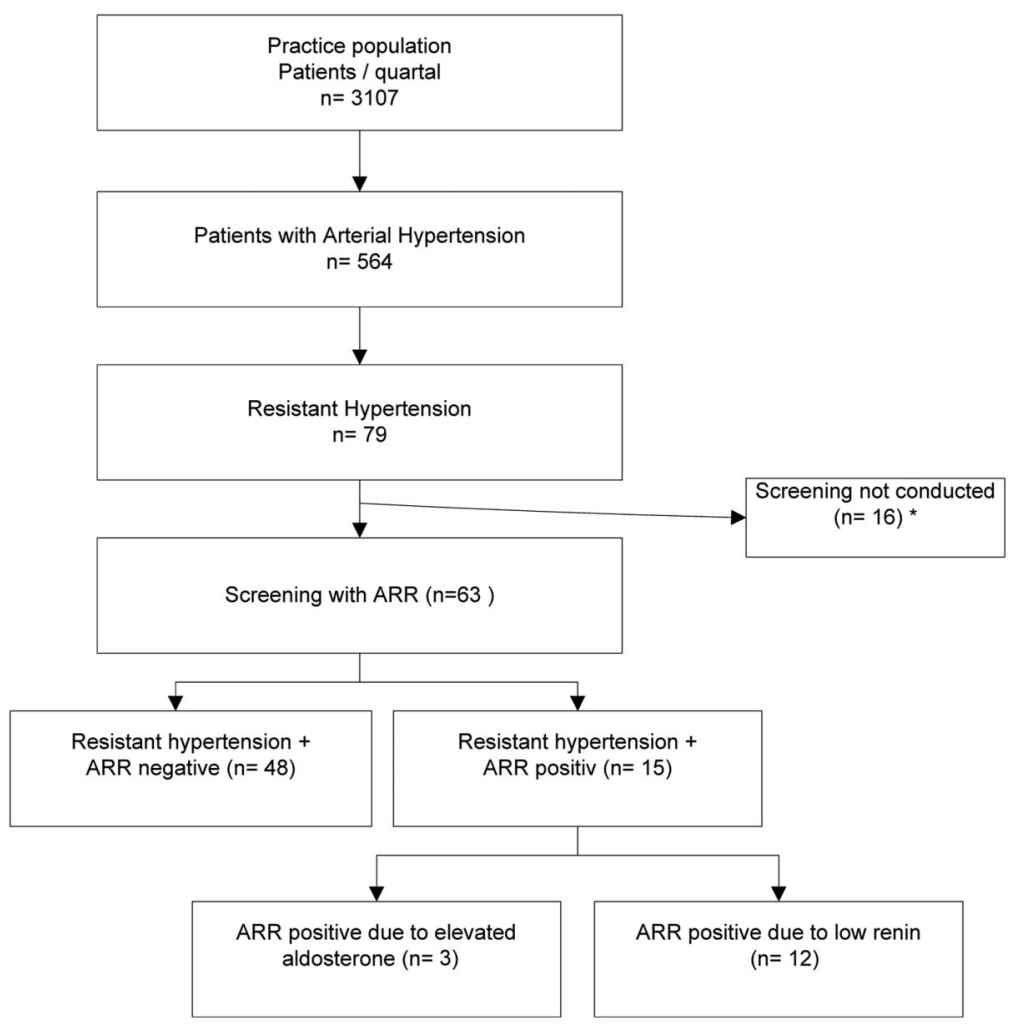


according to the local laboratory, and all measures used radioimmunoassays. Additional analysis included potassium, sodium, body mass index, thyroid-stimulating hormone, and creatinine.

In case of positive test results (ARR $>45$ was considered positive and aldosterone elevated plasma aldosterone concentration $>310 \mathrm{pg} / \mathrm{mL}$ ), patients were referred for confirmation to secondary care. To assess possible barriers and restrictions to screening in our main study, we included a qualitative approach. A focus group session with 3 of the authors (GS, KG, GE) and a group of experienced GPs $(n=8)$ was held, during which the study design was presented and discussed. The discussion was documented by a written protocol. All problematic aspects and possible barriers mentioned were collected and supplemented by documented barriers during the pilot study and regular meetings between participating GPs.

\section{Data Analysis}

Clinical and laboratory data were drawn from patient records. Results were exported into an Access database (Microsoft Corp, Redmond, WA) and data quality was controlled independently by 2 researchers for missing values and consistency of data. For statistical analysis we used R (www.r-project.org); group comparisons were calculated with $t$ test. Laboratory results between the groups were analyzed with Wilcoxon rank sum test because values were not distributed normally. Significance was assumed at $P<.05$ but can only be used for developing hypotheses. We did not adjust significance levels for multiple testing.

To calculate the sample size for main study, we found a sample size of 138 patients is sufficient to support a prevalence rate of $10 \%( \pm 5 \%)$ at the 0.05 significance level with $80 \%$ power.

\section{Results}

Seventy-nine patients with resistant hypertension were included, with a mean age of 69 years $(\mathrm{SD}, \pm$ 10.4 years); $70 \%$ were women. On average, every patient was treated with 3.5 antihypertensive drugs, mainly diuretics ( $\mathrm{n}=61 ; 97 \%)$, angiotensin-converting enzyme inhibitors $(n=58 ; 92 \%)$, and $\beta$ blockers $(n=53 ; 84 \%)$. Further details about the patients are presented in Table 1 . Withdrawal of $\beta$ blockers was possible in 24 of the 53 patients taking them. In the remaining 29 patients, $\beta$ blockers could not be stopped because of patients' clinical conditions, the GP judged it was not appropriate because of comorbidities such as coronary heart disease/cardiac failure, and organizational problems in the practices.

The prevalence of HA (elevated ARR) in unselected primary care patients with a diagnosis of hypertension was 15 of $566(2.65 \%)$. In 63 of 79 patients with resistant hypertension, ARR was measured, revealing an elevated result in $23.8 \%(\mathrm{n}=$ 15). Only in 3 cases this was because of an increased aldosterone level; in the remaining patients, ARR was positive because of low renin levels. Prevalence rates in different subgroups are presented in Table 2. For patient flow, see Figure 1. Age, sex, and current medication as well as laboratory results are presented in Table 1 . Concurrent $\beta$-blocker medication leads to suppression of renin levels $(P=.005)$. There were no significant differences of potassium level between those with or without elevated ARR $(P=.245)$.

Patients with positive ARR due to elevated aldosterone levels were referred for further follow-up as inpatients in a university teaching hospital. Follow-up consisted of confirmation tests (sodium chloride loading test and/or diagnostic imaging) and led to the diagnosis of aldosterone-producing adenomas $(n=2)$ and one case of idiopathic HA. Both patients with aldosterone-producing adenomas underwent uneventful laparoscopic surgery.

\section{Results of Focus Group Discussion}

Discussion with GPs during the focus group disclosed several obstacles against screening for HA in primary care.

\section{Financial}

Laboratory testing is expensive, and high laboratory costs have budget implications for German GPs and may lead to reduced bonus payments for doctors.

\section{Necessity}

GPs are used to dealing with uncertainty in the diagnostic approach. Some of them, therefore, preferred a test and treat strategy (eg, to try spironolactone in patients with resistant hypertension and see what happens). They do not require definite clarification as long as the treatment works and the risk of a potential malignant cause (eg, tumor of the adrenal gland) is very low. 


\begin{tabular}{|c|c|c|c|}
\hline & Resistant Hypertension & Resistant Hypertension and Elevated ARR & $P$ \\
\hline Patients (n) & 63 & 15 & \\
\hline Age (mean years [SD]) & $69.2(10.4)$ & $64.8(12.0)$ & $.208^{*}$ \\
\hline Female (n [\%]) & $44(69.8)$ & $10(66.7)$ & \\
\hline Retired (n [\%]) & $53(84.1)$ & $11(73.3)$ & \\
\hline White (n [\%]) & $100(10.0)$ & $100(10.0)$ & \\
\hline SHI (n [\%]) & $62^{\ddagger}(98.4)$ & $15(100)$ & \\
\hline $\mathrm{BMI}\left(\mathrm{kg} / \mathrm{m}^{2}\right)($ mean $[\mathrm{SD}])$ & $31.3(5.5)$ & $30.9(5.3)$ & $.762^{*}$ \\
\hline ACE inhibitors (n [\%]) & $58(92.1)$ & $13(86.7)$ & \\
\hline $\operatorname{ABA}(\mathrm{n}[\%])$ & $53(84.0)$ & $15(100)$ & \\
\hline CCB (n [\%]) & $37(58.7)$ & $5(33.3)$ & \\
\hline $\operatorname{AAA}(\mathrm{n}[\%])$ & $9(14.3)$ & $3(20.0)$ & \\
\hline Diuretics (n [\%]) & $61(96.8)$ & $14(93.3)$ & \\
\hline Creatinine (mean mg/dl [SD]) & $0.97(0.23)$ & $0.98(0.19)$ & $.816^{*}$ \\
\hline Potassium (mean mEq/1 [SD]) & $4.3(0.47)$ & $4.1(0.52)$ & $.245^{*}$ \\
\hline \multicolumn{4}{|c|}{ Aldosterone (median pg/mL [IQR]) } \\
\hline With $\beta$-blocker & $109(73.0-168.0)(\mathrm{n}=29)$ & $157(116.5-202.5)(\mathrm{n}=11)$ & $.076^{\dagger}$ \\
\hline Without $\beta$-blocker & $108(52.5-140.8)(\mathrm{n}=34)$ & $180(138.2-204.5)(n=4)$ & $.122^{\dagger}$ \\
\hline \multicolumn{4}{|l|}{ Renin (median pg/mL [IQR]) } \\
\hline With $\beta$-blocker & $5.6(3.0-24.1, \mathrm{n}=29)$ & $1.2(1.1-2.2)(\mathrm{n}=11)$ & $<.001^{\dagger}$ \\
\hline Without $\beta$-blocker & $9.0(4.2-29.5, \mathrm{n}=34)$ & $1.0(0.9-1.2)(\mathrm{n}=4)$ & $.005^{\dagger}$ \\
\hline \multicolumn{4}{|l|}{ ARR (median [IQR]) } \\
\hline With $\beta$-blocker & $17.1(6.0-36.5, \mathrm{n}=29)$ & $106.2(68.1-177.5)(\mathrm{n}=11)$ & $<.001^{\dagger}$ \\
\hline Without $\beta$-blocker & $8.9(3.9-24.1, \mathrm{n}=34)$ & $137.1(100.0-186.5)(n=4)$ & $.005^{\dagger}$ \\
\hline
\end{tabular}

\section{Organizational}

Practice software in Germany is not designed for systematic screening. It is not possible to conduct an electronic search for patients with a certain diagnosis in combination with drug therapy (hypertension and 3 drugs). A precondition is electronic access to all prescriptions-this is only possible when GPs record electronically all diagnoses,

Table 2. Prevalence of Hyperaldosteronism (Elevated Aldosterone-Renin Ratio [ARR] and Elevated Aldosterone) in Different Subgroups

\begin{tabular}{lc}
\hline Prevalence of Hyperaldosteronism & Percentage \\
\hline Total patients $(\mathrm{n}=3107)$ & 0.09 \\
Patients with hypertension $(\mathrm{n}=566)$ & 0.53 \\
Patients with resistant hypertension $(\mathrm{n}=79)$ & 3.8 \\
Patients with resistant hypertension and elevated & 20.0 \\
ARR $(\mathrm{n}=15)$ & \\
\hline
\end{tabular}

drugs, and relevant findings such as blood pressure-otherwise a search by hand of all patient records has to be conducted, which is an impossible mission in a busy general practice.

\section{Practical}

Cessation of $\beta$ blockers leads to extra workload (additional practice visits, blood pressure control) and potential clinical risks. It may be inappropriate in patients with cardiac failure, severe arrhythmia, unstable angina, and recent myocardial infarction. In our study, one patient died 9 days after the $\beta$ blocker was discontinued. Autopsy revealed a basilar vein thrombosis as cause of death; even when $\beta$-blocker withdrawal was not linked to the death, this experience leaves the GP with worry about a possible causal association. Finally, blood samples have to be frozen for 
renin measurement, thus creating additional workload for GPs and nurses.

\section{Discussion}

The aims of this pilot study were (1) to assess the prevalence of HA among general practice patients with resistant hypertension and (2) to show possible limitations of systematic screening in primary care. Our results regarding prevalence are in contrast to other studies reporting higher rates (up to 14\%) in unselected hypertensive patients from primary care. ${ }^{10-12}$ The advantage of our study is the rigorous search for all eligible patients in 2 practices with a high standard of electronic documentation, thus avoiding selection bias. We accepted different measures of blood pressure as inclusion criteria, which was necessary because we used clinical data from patient charts as inclusion criteria. Therefore, we had no standardized assessment of blood pressure. Because several measures were taken into account, the risk of patients with a false-positive classification of having resistant hypertension is very low. Within this pilot study we did not have the resources to measure ARR in all hypertensive patients; therefore, it remains unclear how many patients with "normal" hypertension $(<140 / 90 \mathrm{~mm}$ $\mathrm{Hg}$ while taking $\leq 3$ drugs) have an elevated ARR. Our inclusion criteria were in line with guideline recommendations, ${ }^{4}$ but more recent studies have shown high prevalence rates in unselected hypertensive patients in primary care. ${ }^{12}$ Nevertheless, extending a screening of ARR to all hypertensive patients would lead to rising numbers of falsepositive tests with unknown implications in terms of efficiency.

Cessation of a $\beta$ blocker is mandatory to avoid false-positive results because of renin suppression. ${ }^{13}$ This (pre)condition seems to be no problem in clinical trials evaluating ARR, probably because only selected patients were included and intensive follow-up allowed reduction of all drugs that might interfere with ARR. ${ }^{7}$ Outside a clinical trial, $\beta$-blocker cessation turned out to be a relevant obstacle. Therefore, a systematic screening seems to be difficult in general practice and might even present problems for secondary care if all patients with resistant hypertension would be referred for further testing.

We did not aim for confirmatory tests in all patients with elevated ARR, but instead concentrated on those with elevated ARR caused by higher aldosterone levels. This screening routine is recommended $^{13,14}$ to avoid high rates of false-positive results in patients with low renin hypertension.

We might use spironolactone in patients with resistant hypertension without further testing, as some authors suggest, ${ }^{15,16}$ which is a concept advocated by many GPs. On the other hand, our study supports literature findings that demonstrate a benefit of surgical treatment of aldosterone-producing tumors-a therapy with proven cost effectiveness. ${ }^{3}$ Screening recommendations that focus only on aldosterone measurement and that skip the resource-intensive test for renin levels possibly could lead to higher acceptance and feasibility in primary care. At the moment, aldosterone in combination with ARR is recommended ${ }^{17}$ as a screening test, whereas sensitivity of a screening based on aldosterone alone has to be evaluated further.

\section{Conclusions}

The prevalence of HA (elevated ARR) in unselected primary care patients with a diagnosis of resistant hypertension possibly is lower than previously thought. Systematic screening for HA in primary care was not possible in all eligible patients. The main barrier was patients' clinical situation (cardiac failure), which detained their GP from cessation of $\beta$ blockers.

We would like to thank all colleagues who participated in the discussion of the study design. Thanks to Alf Breull for statistical support and to John Rosenblum for linguistic advice.

\section{References}

1. Calhoun DA, Jones D, Textor S, et al. Resistant hypertension: Diagnosis, evaluation, and treatment: a scientific statement from the American Heart Association Professional Education Committee of the Council for High Blood Pressure Research. Circulation 2008;117(25):e510-26.

2. Nishikawa T, Saito J, Omura M. Is primary aldosteronism rare or common among hypertensive patients? Hypertens Res 2007;30(2):103-4.

3. Sywak M, Pasieka JL. Long-term follow-up and cost benefit of adrenalectomy in patients with primary hyperaldosteronism. Br J Surg 2002;89(12):1587-93.

4. Funder JW, Carey RM, Fardella C, et al. Case detection, diagnosis, and treatment of patients with primary aldosteronism: an endocrine society clinical practice guideline. J Clin Endocrinol Metab 2008; 93(9):3266-81.

5. Arlt W. A detour guide to the endocrine society clinical practice guideline on case detection, diagno- 
sis and treatment of patients with primary aldosteronism. Eur J Endocrinol 2010;162(3):435-8.

6. Mosso L, Carvajal C, Gonzalez A, et al. Primary aldosteronism and hypertensive disease. Hypertension 2003;42(2):161-5.

7. Jansen PM, Boomsma F, van den Meiracker AH, Dutch ARRAT Investigators. Aldosterone-to-renin ratio as a screening test for primary aldosteronismthe Dutch ARRAT study. Neth J Med 2008;66(5): 220-8.

8. Douma S, Petidis K, Doumas M, et al. Prevalence of primary hyperaldosteronism in resistant hypertension: a retrospective observational study. Lancet 2008;371(9628):1921-6.

9. Schirpenbach C, Segmiller F, Bidlingmaier M, et al. The diagnosis and treatment of primary hyperaldosteronism in Germany: results on 555 patients from the German Conn Registry. Dtsch Arztebl Int 2009; 106(18):305-11.

10. Fogari R, Preti P, Zoppi A, Rinaldi A, Fogari E, Mugellini A. Prevalence of primary aldosteronism among unselected hypertensive patients: a prospective study based on the use of an aldosterone/renin ratio above 25 as a screening test. Hypertens Res 2007;30(2):111-7.
11. Lim PO, Rodgers P, Cardale K, Watson AD, MacDonald TM. Potentially high prevalence of primary aldosteronism in a primary-care population. Lancet 1999;353(9146):40.

12. Westerdahl C, Bergenfelz A, Isaksson A, Nerbrand C, Valdemarsson S. Primary aldosteronism among newly diagnosed and untreated hypertensive patients in a Swedish primary care area. Scand J Prim Health Care 2011;29(1):57-62.

13. Seiler L, Reincke $M$. The aldosterone to renin ratio in secondary hypertension. Herz 2003;28(8): 686-91.

14. Rossi GP, Seccia TM, Pessina AC. Primary aldosteronism. Part I. Prevalence, screening, and selection of cases for adrenal vein sampling. J Nephrol 2008; 21(4):447-54.

15. Rosendorff C. Spironolactone for all hypertensive patients? J Hypertens 2010;28(1):13-4.

16. Kaplan NM. Deja vu for primary aldosteronism. Lancet 2008;371(9628):1890-1.

17. Rossi GP, Pessina AC, Heagerty AM. Primary aldosteronism: an update on screening, diagnosis and treatment. J Hypertens 2008;26(4):613-21. 